\title{
INVARIANT SUBSPACES FOR ALGEBRAS OF SUBNORMAL OPERATORS. II
}

\author{
JAMES E. THOMSON
}

(Communicated by John B. Conway)

\begin{abstract}
We continue our study of hyperinvariant subspaces for rationally cyclic subnormal operators. We establish a connection between hyperinvariant subspaces and weak-star continuous point evaluations on the commutant.
\end{abstract}

Introduction. Let $K$ be a compact subset of the complex plane $\mathbf{C}$ and let $R(K)$ denote the algebra of rational functions with poles off $K$. For a positive measure $\mu$ with support in $K$ let $R^{2}(K, \mu)$ denote the closure of $R(K)$ in $L^{2}(\mu)$. Every rationally cyclic subnormal operator is unitarily equivalent to multiplication by $z$, $M_{z}$, on an $R^{2}(K, \mu)$-space [1, p. 146]. Under this representation each operator that commutes with $M_{z}$ is represented by multiplication by a function in $R^{2}(K, \mu) \cap$ $L^{\infty}(\mu)$, and conversely [1, p. 147].

In [2] we proved the existence of invariant subspaces for the algebra $R^{2}(K, \mu) \cap$ $L^{\infty}(\mu)$. In this paper we show there exist weak-star continuous point evaluations on $R^{2}(K, \mu) \cap L^{\infty}(\mu)$ if $L^{2}(\mu) \neq R^{2}(K, \mu)$, and we show that these point evaluations give rise to the kinds of invariant subspaces found in [2]. Finally, we establish a connection between analytic bounded point evaluations on $R^{2}$-spaces and $R^{p}$-spaces for $2<p<4$.

Notation. Let $m$ denote area measure on $\mathbf{C}$. For a complex measure $\nu$ with compact support in $\mathbf{C}$ let $\hat{\nu}(z)=\int(\varsigma-z)^{-1} d \nu(\varsigma)$ and $\tilde{\nu}(z)=\int|\varsigma-z|^{-1} d|\nu|(\varsigma)$. By Tonelli's theorem $\tilde{\nu}(z)<\infty m$-a.e. and hence the Cauchy transform $\hat{\nu}$ is defined $m$-a.e. For $g$ in $L^{1}(|\nu|)$ let $\hat{g}$ equal $(g d \nu)^{\wedge}$.

ThEOREM. Let $\bar{g} \in R^{2}(K, \mu)^{\perp}$ and let $A=\{z: \hat{g}(z) \neq 0\}$. Then for m-a.e. $z$ in $A$ there exists a weak-star continuous multiplicative linear functional $e_{z}$ on $R^{2}(K, \mu) \cap L^{\infty}(\mu)$ such that $e_{z}(f)=f(z)$ for each $f$ in $R(K)$. Moreover, for $m$ a.e. such $z$ there exist $x$ and $y$ in $R^{2}(K, \mu)$ such that $e_{z}(f)=\langle f x, y\rangle$ for each $f$ in $R^{2}(K, \mu) \cap L^{\infty}(\mu)$.

REMARK. Let $z$ in $A$ be such that both conclusions of the theorem hold, and let $x$ and $y$ be as in the second conclusion. Let $H$ be the closed linear span of $\left\{(\varsigma-z) f x: f \in R^{2}(K, \mu) \cap L^{\infty}(\mu)\right\}$ in $L^{2}(\mu)$. Since $y \perp H$, it follows that $H$ is a nontrivial hyperinvariant subspace for $M_{\varsigma}$ on $R^{2}(K, \mu)$.

LEMMA 1. Let $p \in(2,4)$ and let $s=2 /(p-2)$. Suppose $f_{n} \in L^{2}(\mu)$ and $\left\|f_{n}\right\|_{2}<2^{-n}$ for each positive integer $n$. Then there exists a function $w: \mathbf{C} \rightarrow(0,1]$ such that $w^{-1} \in L^{s}(\mu), f_{n} \in L^{p}(w d \mu)$ for each $n$, and $f_{n} \rightarrow 0$ in $L^{p}(w d \mu)$.

Received by the editors August 13, 1987.

1980 Mathematics Subject Classification (1985 Revision). Primary 47B20.

Key words and phrases. Subnormal operators, invariant subspaces.

Research supported in part by a grant from the National Science Foundation. 
PROOF. Let $w=\left(1+\sum_{n=1}^{\infty}\left|f_{n}\right|\right)^{2-p}$. Redefine $w$ to be one on the set where the infinite series diverges. Since $w^{-s}=\left(1+\sum\left|f_{n}\right|\right)^{2}$, it follows that $w^{-s} \in L^{1}(\mu)$ and hence $w^{-1} \in L^{s}(\mu)$. Finally, $\left|f_{n}\right|^{p} w \leq\left|f_{n}\right|^{2}$ implies that $f_{n} \in L^{p}(w d \mu)$ for each $n$ and that $f_{n} \rightarrow 0$ in $L^{p}(w d \mu)$.

LEMMA 2. Let $p \in(2,4), s=2 /(p-2)$, and $q=p(p-1)^{-1}$. Suppose $R^{2}(K, \mu) \neq L^{2}(\mu)$. Let $f_{1}, f_{2} \in R^{2}(K, \mu)$. Then there exists a function $w: \mathbf{C} \rightarrow$ $(0,1]$ such that $w^{-1} \in L^{s}(\mu), R^{p}(K, w d \mu) \neq L^{p}(w d \mu)$, and $f_{1}, f_{2} \in R^{p}(K, w d \mu)$.

ProOF. Define $f_{n}$ to be $f_{1}$ when $n$ is odd and $f_{2}$ when $n$ is even. Let $\left\{r_{n}\right\}$ be a sequence of functions from $R(K)$ such that $\left\|r_{n}-f_{n}\right\|_{2}<2^{-n}$ for each $n$. Applying Lemma 1 to the sequence $\left\{r_{n}-f_{n}\right\}$, we obtain a function $w: \mathbf{C} \rightarrow(0,1]$ such that $w^{-1} \in L^{s}(\mu)$ and $r_{n}-f_{n} \rightarrow 0$ in $L^{p}(w d \mu)$. It follows immediately that $f_{1}$ and $f_{2}$ are in $R^{p}(K, w d \mu)$. If $\bar{g} \in R^{2}(K, \mu)^{\perp}$ then $(g / w) \in L^{q}(w d \mu)$ by Hölder's inequality. Since $R^{2}(K, \mu) \neq L^{2}(\mu)$, it follows that $R^{p}(K, w d \mu) \neq L^{p}(w d \mu)$.

PROOF OF THE THEOREM. Fix $p$ and $q$ as in Lemma 2 . Let $B$ be a compact subset of $A$ such that the following hold:

(i) $\left.(g d \mu)^{\sim}\right|_{B}$ is continuous;

(ii) $(\varsigma-z)^{-1} g(\varsigma) \in L^{q}(\mu)$ for each $z \in B$.

As a consequence of (i), $\left.\hat{g}\right|_{B}$ is continuous and hence $\hat{g}$ is bounded away from zero on $B$. Let $E$ be the set of points in $B$ that are of full density with respect to $m$. We shall show that both conclusions of the theorem hold for each $z$ in $E$. We leave it as an exercise that $m$-a.e. $z$ in $A$ belongs to such an $E$.

For $z \in E$ let $e_{z}(f)=\hat{g}(z)^{-1} \int(\varsigma-z)^{-1} f(\varsigma) g(\varsigma) d \mu(\varsigma)$ for each $f \in R^{2}(K, \mu) \cap$ $L^{\infty}(\mu)$. It is easy to check that $e_{z}(f)=f(z)$ if $f \in R(K)$. By the main proof in [2] there exist $x \in R^{2}(K, \mu)$ and $y \in L^{2}(\mu)$ such that $e_{z}(f)=\langle f x, y\rangle$ for each $f$ in $R^{2}(K, \mu) \cap L^{\infty}(\mu)$. Projecting $y$ into $R^{2}(K, \mu)$, we may assume that $y \in R^{2}(K, \mu)$. It remains to show that $e_{z}$ is multiplicative on $R^{2}(K, \mu) \cap L^{\infty}(\mu)$.

For $f \in R^{2}(K, \mu) \cap L^{\infty}(\mu)$ the map $z \rightarrow e_{z}(f)$ is continuous on $E$. This is a consequence of assumption (i). Thus it suffices to show that for $f_{1}, f_{2} \in R^{2}(K, \mu) \cap$ $L^{\infty}(\mu)$ there exists a dense subset $F$ of $E$ such that $e_{z}\left(f_{1} f_{2}\right)=e_{z}\left(f_{1}\right) e_{z}\left(f_{2}\right)$ for each $z \in F$. Let $F$ be the set of $z \in E$ for which

$$
\int|\varsigma-z|^{-q}|(g / w)|^{q} w d \mu<\infty
$$

By Tonelli's theorem $m(E \backslash F)=0$. For $z \in F$ the map $f \rightarrow e_{z}(f)$ is $L^{p}(w d \mu)$ continuous on $R(K)$. Since $e_{z}$ is multiplicative on $R(K)$, it follows from taking $L^{p}(w d \mu)$ limits that $e_{z}\left(f_{1} f\right)=e_{z}\left(f_{1}\right) e_{z}(f)$ for each $f \in R(K)$. Taking $L^{p}(w d \mu)$ limits again, we obtain $e_{z}\left(f_{1} f_{2}\right)=e_{z}\left(f_{1}\right) e_{z}\left(f_{2}\right)$. That does it.

REMARK. Let $P^{r}(\mu)$ denote the closure of the polynomials in $L^{r}(\mu)$. Suppose $P^{2}(\mu)$ has no analytic bounded point evaluations. Then there exists a function $f$ in $P^{2}(\mu)$ that is essentially unbounded on every relatively open subset of the support of $\mu$. If $P^{2}(\mu) \neq L^{2}(\mu)$ then by Lemma $2 f$ is in a nontrivial $P^{r}$-space for every $r \in(2,4)$. It follows that none of those $P^{r}$-spaces has any analytic bounded point evaluations. Thus, to show that every nontrivial $P^{2}$-space has some analytic bounded point evaluations, it suffices to show that every nontrivial $P^{r}$-space has analytic bounded point evaluations for some $r \in(2,4)$. It is true that bounded point evaluations exist for every nontrivial $P^{r}$-space for $r>2$. 


\section{REFERENCES}

1. J. B. Conway, Subnormal operators, Pitman, Boston, Mass., 1981.

2. J. E. Thomson, Invariant subspaces for algebras of subnormal operators, Proc. Amer. Math. Soc. 96 (1985), 462-464.

Department of Mathematics, Virginia Polytechnic institute and State UNIVERSITY, BLACKSBURG, VIRGINIA 24061 